\title{
BeppoSAX discovery of the X-ray afterglow of GRB 971227
}

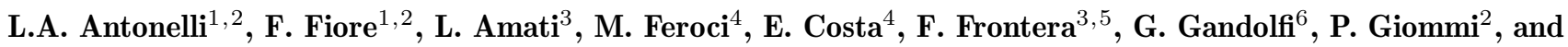 \\ L. Piro ${ }^{4}$ \\ 1 Osservatorio Astronomico di Roma, Via Frascati 33, I-00040 Monte Porzio Catone (RM), Italy \\ 2 BeppoSAX Science Data Center, Via Corcolle 19, I-00131 Roma, Italy \\ 3 Istituto Tecnologie e Studio Radiazioni Extraterrestri, CNR, Via Gobetti 101, I-40129 Bologna, Italy \\ 4 Istituto di Astrofisica Spaziale, CNR, Via Fosso del Cavaliere, I-00133 Roma, Italy \\ 5 Dipartimento di Fisica, Università di Ferrara, Via Paradiso 11, I-44100 Ferrara, Italy \\ 6 BeppoSAX Scientific Operation Center, Telespazio, Via Corcolle 19, I-00131 Roma, Italy
}

Received March 16; accepted April 22, 1999

\begin{abstract}
The BeppoSAX Narrow Field Instruments observed the region of sky containing GRB 97122714 hours after the initial burst. A faint source (1SAXJ1257.3+5924) is detected in a position consistent with the BeppoSAX Wide Field Camera position, and disappears during the second half of the observation. In contrast to some other GRBs observed by BeppoSAX no optical and radio afterglows were unambiguously observed.
\end{abstract}

Key words: gamma-rays: bursts; X-rays: observation

\section{Introduction}

On 199727 December, 08:43:59.99 UT a gamma ray burst was detected by BATSE (Woods et al. 1997) and by BeppoSAX GRBM and WFC unit 2 (Coletta et al. 1997). The duration of the event was about $7 \mathrm{~s}$ and the peak flux measured by BATSE was $(3.3 \pm 0.2)$ photons $\mathrm{cm}^{-2} \mathrm{~s}^{-1}$. The total fluence (over $7 \mathrm{~s}$ ) was $(9.3 \pm 1.4) 10^{-7} \mathrm{erg} \mathrm{cm}^{-2} \mathrm{~s}^{-1}$ in the $(20-300) \mathrm{keV}$ energy band and no signal has been detected above $300 \mathrm{keV}$. It was localized in the WFC2 field of view and imaged with BeppoSAX Narrow Field Instruments about 14 hours after the burst. This observation shows a faint X-Ray source visible in the first $11600 \mathrm{~s}$ of the observation only. The "Original" BATSE error box was observed by the Livermore Optical Transient Imaging System (LOTIS) about $14 \mathrm{~s}$ after the burst but the analysis of LOTIS images does not show any optical transient within the BeppoSAX WFC error box brighter than $R=14.2$ mag (Park 1997; Williams et al. 1999). The BeppoSAX WFC error box was imaged by many

Send offprint requests to: angelo@quasar.mporzio.astro.it ground based telescopes. A claim of a detection came from an image taken at the $2.2 \mathrm{~m}$ CAHA telescope. They observed the GRB field about 13.7 hours after the burst and detected a source with $R=19.5$ mag (Castro-Tirado et al. 1997) within the $1.5^{\prime}$ BeppoSAX NFI error circle (Piro et al. 1997). Further observations in the optical band did not show any significantly detection (CastroTirado et al. 1997; Groot et al. 1997; Ramaprakash et al. 1997; Bartolini et al. 1997, 1999). Deep images taken with the Keck-II about 3 and 5 days after the burst did not show any object brighter than $R=23$ mag showing significantly variation by more than $0.2 \mathrm{mag}$.

\section{Data analysis and results}

This burst was detected by only one lateral shield of the GRBM on-board BeppoSAX. The event shows a single peak structure lasting about $7 \mathrm{~s}$ (Coletta et al. 1997) and the peak intensity was $(2.6 \pm 0.3)$ photons $\mathrm{cm}^{-2} \mathrm{~s}^{-1}$ in the energy range $40-700 \mathrm{keV}$. The 256 -channel GRBM data permits a sensitive spectral analysis with a $128 \mathrm{~s}$ accumulation interval. Fitting GRBM data with a single power law we found a photon index of $\Gamma=-2.58 \pm 0.33$. The fluence is $(6.6 \pm 0.7) 10^{-7} \mathrm{erg} \mathrm{cm}^{-2}$ in the $(40-700) \mathrm{keV}$ band and $(9.5 \pm 0.9) 10^{-7} \mathrm{erg} \mathrm{cm}^{-2}$ in the $(20-300) \mathrm{keV}$. Assuming as a spectral model a broken power law with energy cut-off above $300 \mathrm{keV}$ (BATSE results) we found $\Gamma_{1}=-2.30 \pm 0.43, E_{\text {cut }- \text { off }}=155 \pm 1 \mathrm{keV}$ and $\Gamma_{2} \leq-9$ $\left(\chi_{r}^{2}=1.3\right)$. The GRBM results are consistent with results from BATSE and show evidence of a quite soft $\gamma$-ray emission.

This GRB was also detected by the WFC unit 2, with a similar time profile structure and about the same duration. The $2-10 \mathrm{keV}$ peak flux was $3.610^{-8} \mathrm{erg} \mathrm{cm}^{-2}$ $\mathrm{s}^{-1}$ (Coletta et al. 1997) a $2-10 \mathrm{keV}$ fluence of 1.0 $10^{-7} \mathrm{erg} \mathrm{cm}^{-2}$ and a mean flux, in the same energy band, 
of $1.410^{-8} \mathrm{erg} \mathrm{cm}^{-2} \mathrm{~s}^{-1}$. The position given by the quicklook analysis was R.A. $=12^{\mathrm{h}} 57^{\mathrm{m}} 29^{\mathrm{s}}$, Dec. $=+59^{\circ} 16.3$ (equinox 2000.0). The event was detected in a part of the orbit when the satellite was not in an optimal pointing configuration, resulting in an estimated error radius of 10 arcmin.

The $10^{\prime}$ error box of GRB 971227 was imaged with the narrow field X-ray instruments (NFI) on-board BeppoSAX as a Target of Opportunity observation. The GRB followup observation started $51960 \mathrm{~s}$ after the trigger time, from 27 December 22:49 U.T. to 28 December 18:06 U.T., for a total net exposure time of $37000 \mathrm{~s}$ with the MECS and $16316 \mathrm{~s}$ with the LECS (the latter being operated only during satellite night-time).

Two faint X-ray sources were detected in the WFC error box by the BeppoSAX NFI (Piro et al. 1997). It is worth noting that other faint sources may be present in the error circle and they could be hidden by the detector strongback. The source closer to the center of the field, 1SAX J1257.5+5915, has a measured count rate of $(1.6 \pm 0.4) 10^{-3}$ cts s $^{-1}$ corresponding to a flux of $1.110^{-13} \mathrm{erg} \mathrm{cm}^{-2} \mathrm{~s}^{-1}$. All fluxes quoted in this paper are calculated assuming a spectrum described by an absorbed power law with the galactic value for the absorption and $\Gamma=2.1$. This source does not show a fading behaviour and may be a field source. We expect, from the BeppoSAX $\log N-\log S$ (Giommi et al. 1998), to find in the $2-10 \mathrm{keV}$ band about 20 sources per squaredegree at the measured flux: so, in the WFC error box, we expect to find 1.7 sources. The other source, 1SAX J1257.3+5924, showed, during the NFI observation, a fading behaviour making it the best candidate for the X-ray afterglow. We divided the total observation in two parts and the source counts varied from $(3.6 \pm 1.0) 10^{-3} \mathrm{cts} \mathrm{s}^{-1}$ measured in the first $11555 \mathrm{~s}$ to $\left(2.9310^{-3} \operatorname{cts~s}^{-1}\right.$ ( $3 \sigma$ U.L.) in the last $23784 \mathrm{~s}$. The corresponding fluxes varied from 2.6 $10^{-13} \mathrm{erg} \mathrm{cm}^{-2} \mathrm{~s}^{-1}$ to $2.11^{-13} \mathrm{erg} \mathrm{cm}^{-2} \mathrm{~s}^{-1}$ (3 $\sigma$ upper limit). These values together with the WFC value show a decaying law $F(t) \propto t^{-\alpha}$ with $\alpha=1.12_{-0.05}^{+0.08}$ (see figure). The refined position of this source is R.A. $=12^{\mathrm{h}}$ $57^{\mathrm{m}} 17.2^{\mathrm{s}}$ and Dec. $=+59^{\circ} 24^{\prime} 02^{\prime}$ (equinox 2000.0) with an error circle of 1.5 arcmin due to the poor statistic.

\section{Discussion and conclusions}

GRB 971227 is one of the most puzzling GRB observed by BeppoSAX. We associate the fading X-ray source, 1SAX J1257.3+5924, detected in the BeppoSAX MECS, with the best candidate for the X-ray afterglow of GRB 971227. This X-ray afterglow shows a slow decay behaviour tipical of that afterglows discovered both in optical and in X-rays (e.g. GRB 970228, GRB 970508, etc.). On the contrary no optical counterpart was unambiguosly discovered for this GBR as in the case of GRB showing a faster decay (e.g. GRB 970111 (Feroci et al. 1998), GRB 970402 (Nicastro et al. 1998) etc.). The fact that X-ray, optical

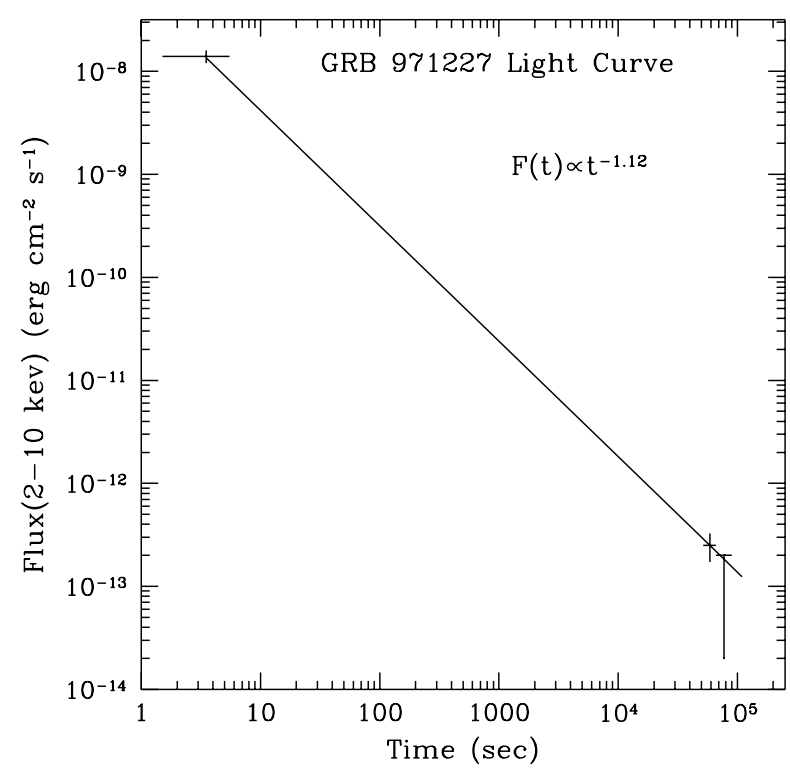

Fig. 1. X-ray $(2-10 \mathrm{keV})$ decay law of the candidate counterpart of GRB 971227. The power-law index, 1.12, needed for connecting the WFC GRB 971227 mean flux and the 1SAXJ $1257.2+5924$ flux

and radio afterglow are not always observed pointing toward a GRB region is currently explained invoking environment effects. If the optical detection of CastroTirado and collaborators is true the optical decay should be faster than that observed at higher energies. This result is different from any other observed in the GRB afterglows up to now and is not easily explainable in terms of environment effects.

Acknowledgements. This research is supported by the Italian Space Agency (ASI). All authors warmly thank the teams of the BeppoSAX Scientific Operation Center, Operation Control Center and Science Data Center for their support to the GRB program.

\section{References}

Bartolini C., et al., 1997, GCN, No. 20

Bartolini C., et al., 1999 (this volume)

Castro-Tirado A.J., et al., 1997, IAU Circ., No. 6800

Coletta A., et al., 1997, IAU Circ., No. 6796

Giommi P., Fiore F., Ricci D., et al., 1998, in Proceedings of "The Active X-Ray Sky", Scarsi L., Bradt H., Giommi P., Fiore F. (eds.). Elsevier Science B.V., p. 591

Groot P., et al., 1997, GCN Circ., No. 17

Park H.S., et al., 1997, GCN Circ., No. 19

Piro L., et al., 1997, IAU Circ., No. 6797

Ramaprakash A.N., et al., 1997/GCN Circ., No. 24

Williams G.G., Park H.S., Ables E., et al., 1999, astroph/9902190

Woods P.M., et al., 1997, IAU Circ., No. 6798 